\title{
Riesz Bases of Root Vectors of Indefinite Sturm-Liouville Problems with Eigenparameter Dependent Boundary Conditions, I
}

\author{
Paul Binding and Branko Ćurgus

\begin{abstract}
We consider a regular indefinite Sturm-Liouville problem with two self-adjoint boundary conditions, one being affinely dependent on the eigenparameter. We give sufficient conditions under which a basis of each root subspace for this Sturm-Liouville problem can be selected so that the union of all these bases constitutes a Riesz basis of a corresponding weighted Hilbert space.
\end{abstract}

Mathematics Subject Classification (2000). Primary 34L10, 34B24, 34B09, 47B50.

Keywords. Sturm-Liouville equations, indefinite weight functions, Riesz bases.

\section{Introduction}

We consider a regular indefinite Sturm-Liouville boundary eigenvalue problem of the form

$$
-\left(p f^{\prime}\right)^{\prime}+q f=\lambda r f \quad \text { on } \quad[-1,1] .
$$

The coefficients $1 / p, q, r$ in (1.1) are assumed to be real and integrable over $[-1,1]$, $p(x)>0$, and $x r(x)>0$ for almost all $x \in[-1,1]$. We impose two boundary conditions on (1.1) (only one of which is $\lambda$-dependent):

$$
\mathbf{L} \mathbf{b}(f)=0, \quad \operatorname{Mb}(f)=\lambda \mathbf{N b}(f) .
$$

where $\mathrm{L}, \mathrm{M}$ and $\mathrm{N}$ are $1 \times 4$ nonzero (row) matrices and the boundary mapping $\mathbf{b}$ is defined for all $f$ in the domain of (1.1) by

$$
\mathbf{b}(f)=\left[\begin{array}{llll}
f(-1) & f(1) & \left(p f^{\prime}\right)(-1) & \left(p f^{\prime}\right)(1)
\end{array}\right]^{T} .
$$


We shall utilize an operator theoretic framework developed in 3 . Under Condition 2.1 below, a self-adjoint operator $A$ in the Krein space $L_{2, r}(-1,1) \oplus \mathbb{C}_{\Delta}$ can be associated with the eigenvalue problem (1.1), (1.2). Here $\Delta$ is a nonzero real number which is determined by $\mathrm{M}$ and $\mathrm{N}$ - see Section 2 for details. We remark that the topology of this Krein space is that of the corresponding Hilbert space $L_{2,|r|}(-1,1) \oplus \mathbb{C}_{|\Delta|}$. (In the rest of the paper we abbreviate $L_{2, r}(-1,1)$ to $L_{2, r}$ and $L_{2,|r|}(-1,1)$ to $L_{2,|r|}$.) Our main goal in this paper is to provide sufficient conditions on the coefficients in (1.1), (1.2) under which there is a Riesz basis of the above Hilbert space consisting of the union of bases for all the root subspaces of the above operator $A$. This will be referred to for the remainder of this section as the Riesz-basis property of $A$.

Completeness and expansion theorems with a stronger topology, but in a smaller space corresponding to the form domain of the operator $A$, have been considered by many authors - see [3] (and the references there) and 12. Although the topology of the Krein space $L_{2, r} \oplus \mathbb{C}_{\Delta}$ is weaker than the topology of the form domain, which in our case is a Pontryagin space, the expansion question turns out to be much more challenging mathematically.

Indeed, even for the case when the boundary conditions are $\lambda$-independent this problem is nontrivial. In our notation, this case corresponds to $L$ being a nonsingular $2 \times 4$ matrix, with the second equation in (1.2) suppressed. The Rieszbasis property of the operator corresponding to $A$, now defined in $L_{2, r}$, has been discussed by several authors, e.g., in [2, 6, 9, 14, 15. The first general sufficient condition for this was given by Beals [2, who required the weight function $r$ to behave like a power of the independent variable $x$ in an open neighborhood of the turning point $x=0$, although his method does allow more general weight functions. Refinements of Beals's method in [9] and [15] show that a "one-sided" condition on $r$ (i.e., in only a half-neighborhood of $x=0$ ) is enough to guarantee the Rieszbasis property. That some extra condition on $r$ is indeed necessary follows from [15] where Volkmer showed that weight functions $r$ exist for which the corresponding Sturm-Liouville problem (1.1), under the conditions used here, does not have the Riesz-basis property. Explicit examples of such weight functions were given in [1] and [10. Recently, Parfyonov 13 has given an explicit necessary and sufficient condition for the Riesz basis property in the case $p=1, q=0$ with odd weight function $r$. Here, and in most of the above references, Dirichlet boundary conditions were imposed.

General self-adjoint (perhaps non-separated, but still $\lambda$-independent) boundary conditions were treated by Ćurgus and Langer [6]. They showed that if the essential boundary conditions, i.e., those not including derivatives, were separated, then a Beals-type condition in a neighborhood of $x=0$ was sufficient for the Riesz-basis property. But if some of the non-separated boundary conditions were essential then [6] established the Riesz-basis property only by imposing extra restrictions on the weight function in (half-)neighborhoods of both endpoints of the interval $[-1,1]$. Again, some extra restriction is necessary, since in [4] we gave an 
explicit example of (1.1) under the conditions used here, satisfying a Beals-type condition at $x=0$, but without the Riesz-basis property. Of course at least one (in fact one, in this antiperiodic case) boundary condition was essential and nonseparated. In some sense, then, the boundary \pm 1 behaves as a turning point under such boundary conditions.

In summary, the Riesz-basis property is quite subtle, and depends significantly on the nature of the boundary conditions even when they are independent of $\lambda$. In this paper and its sequel, we shall examine the analogous situation for the cases of one and two $\lambda$-dependent boundary conditions, where the possibilities for the $(\lambda$-dependent) boundary conditions are much greater. As in the $\lambda$-independent case, a condition on the weight function is needed near the turning point $x=0$ to ensure the Riesz-basis property of $A$. We shall develop such a condition (which is implied by the ones discussed above) in Section 4 Depending on the nature of the boundary conditions (1.2), we may also need a condition near the boundary, and this is discussed in Section 5. It should be remarked that for the case of exactly one $\lambda$-dependent boundary condition treated here we need only one such condition, near either $x=-1$ or $x=1$, and this can be viewed as a "one-sided" condition at \pm 1 . In the case of two $\lambda$-dependent boundary conditions we shall also need a condition involving both boundary points $x=-1$ and $x=1$.

It turns out that all the above conditions have a common core. This is not immediately obvious, since there are differences between the "turning points" 0 and \pm 1 . For example, when the boundary conditions are separated, the values of $f$ and $f^{\prime}$ are equal at 0 but are independent at -1 and 1 . The common core, which will also be needed in Part II, involves the notion of smoothly connected half-neighborhoods, and this is defined and studied in Section 3

In order to apply the above conditions, we use a criterion in Theorem 2.2 , equivalent to the Riesz-basis property of $A$, involving a positive homeomorphism of $L_{2, r} \oplus \mathbb{C}_{\Delta}$ with the form domain of $A$ as an invariant subspace. This, together with certain mollification arguments, is used for our main results, which are detailed in Section 6. To paraphrase these, we recall that a $\lambda$-independent boundary condition is essential if it does not include derivatives. Similarly, a $\lambda$-dependent boundary condition will be called essential if it does not include derivatives in the $\lambda$-terms.

In Theorem 6.1 we discuss situations when a condition on $r$ near $x=0$ suffices for the Riesz-basis property of $A$. For example this holds when the first ( $\lambda$-independent) boundary condition in (1.2) is either non-essential, or essential and separated, and the second ( $\lambda$-dependent) one is non-essential. If the latter condition is essential instead, then the same result holds if a sign condition is also satisfied, and this includes a result of Fleige [11], which is the only reference we know where the Riesz-basis property of $A$ has been studied for $\lambda$-dependent boundary conditions.

In Theorems 6.2 and 6.3 we consider those cases of (1.2) which are not covered by Theorem 6.1. Then we require a condition near just one of the boundary points \pm 1 , not both as in [6]. The choice of the boundary point is arbitrary in Theorem6.2 which deals with the case when the boundary conditions in (1.2) are, respectively, 
essential non-separated and non-essential. In Theorem 6.3, however, this choice is not arbitrary but depends on the sign of the number $\Delta$ used in defining the inner product on $L_{2, r} \oplus \mathbb{C}_{\Delta}$.

\section{Operators associated with the eigenvalue problem}

The maximal operator $S_{\max }$ in $L_{2, r}(-1,1)=L_{2, r}$ associated with (1.1) is defined by

$$
S_{\max }: f \mapsto \ell(f):=\frac{1}{r}\left(-\left(p f^{\prime}\right)^{\prime}+q f\right), \quad f \in \mathcal{D}\left(S_{\max }\right),
$$

where

$$
\mathcal{D}\left(S_{\max }\right)=\mathcal{D}_{\max }=\left\{f \in L_{2, r}: f, p f^{\prime} \in A C[0,1], \ell(f) \in L_{2, r}\right\} .
$$

We define the boundary mapping $\mathbf{b}$ by

$$
\mathbf{b}(f):=\left[\begin{array}{llll}
f(-1) & f(1) & \left(p f^{\prime}\right)(-1) & \left(p f^{\prime}\right)(1)
\end{array}\right]^{T}, \quad f \in \mathcal{D}\left(S_{\max }\right),
$$

and the concomitant matrix $\mathbf{Q}$ corresponding to $\mathbf{b}$ by

$$
\mathrm{Q}:=i\left[\begin{array}{cccc}
0 & 0 & -1 & 0 \\
0 & 0 & 0 & 1 \\
1 & 0 & 0 & 0 \\
0 & -1 & 0 & 0
\end{array}\right] .
$$

We notice that $Q=Q^{-1}$. Integrating by parts we easily calculate that

$$
\int_{-1}^{1} S_{\max } f \bar{g} r-\int_{-1}^{1} f S_{\max } \bar{g} r=i \mathbf{b}(g)^{*} \mathrm{Qb}(f), \quad f, g \in \mathcal{D}\left(S_{\max }\right) .
$$

Throughout, we shall impose the following nondegeneracy and self-adjointness conditions on the boundary data.

Condition 2.1. The row vectors L, M and N in (1.2) satisfy:

(1) the $3 \times 4$ matrix $\left[\begin{array}{c}\mathrm{L} \\ \mathrm{M} \\ \mathrm{N}\end{array}\right]$ has rank 3,

(2) $\mathrm{LQL}^{*}=\mathrm{MQM}^{*}=\mathrm{NQN}^{*}=\mathrm{LQM}^{*}=\mathrm{LQN}^{*}=0$,

(3) $i \mathrm{MQ}^{-1} \mathrm{~N}^{*}$ is a nonzero real number and we define

$$
\Delta=-\frac{i}{\mathrm{MQ}^{-1} \mathrm{~N}^{*}} \text {. }
$$

Clearly the boundary value problem (1.1)-(1.2) will not change if row reduction is applied to the coefficient matrix

$$
\left[\begin{array}{cc}
L & 0 \\
M & N
\end{array}\right]
$$


In what follows we will assume that the $2 \times 8$ matrix in (2.2) is row reduced to row echelon form (starting the reduction at the bottom right corner). After the row reduction, we write the row vectors $\mathrm{L}$ and $\mathrm{N}$ as

$$
\mathrm{L}=\left[\begin{array}{ll}
\mathrm{L}_{e} & \mathrm{~L}_{n}
\end{array}\right], \quad \mathrm{N}=\left[\begin{array}{ll}
\mathrm{N}_{e} & \mathrm{~N}_{n}
\end{array}\right] \text {. }
$$

If either of the $1 \times 2$ matrices $\mathrm{L}_{n}, \mathrm{~N}_{n}$ is nonzero, the corresponding boundary condition is called "non-essential". In any case these matrices do not appear in the representation of the form domain of $A$, discussed below, but they will play an important role in our conditions for Riesz bases in Section 6. The $1 \times 2$ matrices $\mathrm{L}_{e}$ and $\mathrm{N}_{e}$ represent the "essential" boundary conditions if the non-essential parts $\mathrm{L}_{n}$ and $\mathrm{N}_{n}$ are zero matrices.

Next we define a Krein space operator associated with the problem (1.1)(1.2). We consider the linear space $L_{2,|r|} \oplus \mathbb{C}$, equipped with the inner product

$$
\left[\left(\begin{array}{l}
f \\
z
\end{array}\right),\left(\begin{array}{c}
g \\
w
\end{array}\right)\right]:=\int_{-1}^{1} f \bar{g} r+\bar{w} \Delta z, \quad f, g \in L_{2,|r|}, z, w \in \mathbb{C} .
$$

Then $\left(L_{2,|r|} \oplus \mathbb{C},[\cdot, \cdot]\right)$ is a Krein space, which we denote by $L_{2, r} \oplus \mathbb{C}_{\Delta}$. A fundamental symmetry on this Krein space is given by

$$
J:=\left[\begin{array}{cc}
J_{0} & 0 \\
0 & \operatorname{sgn} \Delta
\end{array}\right]
$$

where $\operatorname{sgn} \Delta \in\{-1,1\}$ and $J_{0}: L_{2, r} \rightarrow L_{2, r}$ is defined by

$$
\left(J_{0} f\right)(x):=f(x) \operatorname{sgn}(r(x)), \quad x \in[-1,1] .
$$

Then $[\mathrm{J} \cdot, \cdot]$ is a positive definite inner product which turns $L_{2, r} \oplus \mathbb{C}_{\Delta}$ into a Hilbert space $L_{2,|r|} \oplus \mathbb{C}_{|\Delta|}$. The topology of $L_{2, r} \oplus \mathbb{C}_{\Delta}$ is defined to be that of $L_{2,|r|} \oplus \mathbb{C}_{|\Delta|}$, and a Riesz basis of $L_{2, r} \oplus \mathbb{C}_{\Delta}$ is defined as a homeomorphic image of an orthonormal basis of $L_{2,|r|} \oplus \mathbb{C}_{|\Delta|}$.

We define the operator $A$ in the Krein space $L_{2, r} \oplus \mathbb{C}_{\Delta}$ on the domain

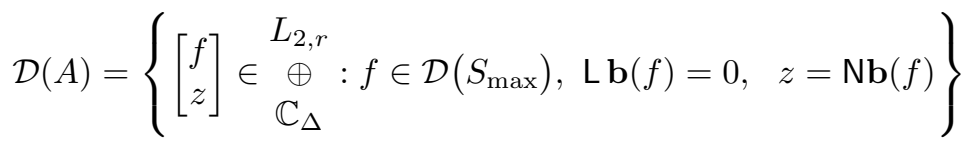

by

$$
A\left[\begin{array}{c}
f \\
\mathrm{Nb}(f)
\end{array}\right]:=\left[\begin{array}{c}
S_{\max } f \\
\mathrm{Mb}(f)
\end{array}\right], \quad\left[\begin{array}{c}
f \\
\mathrm{Nb}(f)
\end{array}\right] \in \mathcal{D}(A) .
$$

Using [3, Theorems 3.3 and 4.1] we see that this operator is self-adjoint in $L_{2, r} \oplus \mathbb{C}_{\Delta}$ and in particular:

(i) $A$ is quasi-uniformly positive [7] (and therefore definitizable) in $L_{2, r} \oplus \mathbb{C}_{\Delta}$.

(ii) $A$ has a discrete spectrum.

(iii) The root subspaces corresponding to real distinct eigenvalues of $A$ are mutually orthogonal in the Krein space $L_{2, r} \oplus \mathbb{C}_{\Delta}$.

(iv) All but finitely many eigenvalues of $A$ are semisimple and real. 
For further properties of $A$, we refer the reader to [3, Theorem 3.3]. From (i), (ii) and the characterization of the regularity of the critical point infinity for definitizable operators in Krein spaces given in [5, Theorem 3.2], we then obtain the following, which is our central tool.

Theorem 2.2. Let $\mathcal{F}(A)$ denote the form domain of $A$. There exists a basis for each root subspace of $A$, so that the union of all these bases is a Riesz basis of $L_{2,|r|} \oplus \mathbb{C}_{|\Delta|}$ if and only if there exists a bounded, boundedly invertible, positive operator $W$ in $L_{2, r} \oplus \mathbb{C}_{\Delta}$ such that $W \mathcal{F}(A) \subset \mathcal{F}(A)$.

In order to apply this result, we need to characterize the form domain $\mathcal{F}(A)$. To this end, let $\mathcal{F}_{\max }$ be the set of all functions $f$ in $L_{2, r}$, absolutely continuous on $[-1,1]$, such that $\int_{-1}^{1} p\left|f^{\prime}\right|^{2}<+\infty$. On $\mathcal{F}_{\max }$ we define the essential boundary mapping $\mathbf{b}_{e}: \mathcal{F}_{\max } \rightarrow \mathbb{C}^{2}$ by

$$
\mathbf{b}_{e}(f):=\left[\begin{array}{ll}
f(-1) & f(1)
\end{array}\right]^{T}, \quad f \in \mathcal{F}_{\max } .
$$

Clearly $\mathbf{b}_{e}$ is surjective.

By [3, Theorem 4.2], there are four possible cases for the form domain $\mathcal{F}(A)$ of $A$ : If $\mathrm{L}_{n} \neq 0$ and $\mathrm{N}_{n} \neq 0$, then

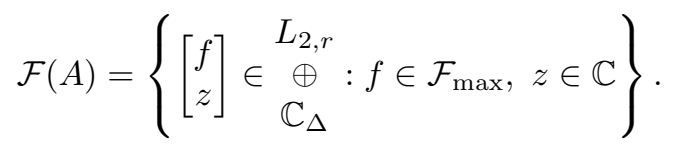

If $\mathrm{L}_{n}=0$ and $\mathrm{N}_{n} \neq 0$, then

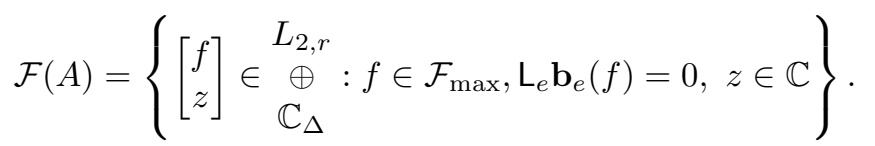

If $\mathrm{L}_{n} \neq 0$ and $\mathrm{N}_{n}=0$, then

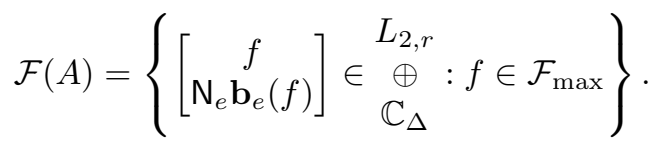

If $\mathrm{L}_{n}=0$ and $\mathrm{N}_{n}=0$, then

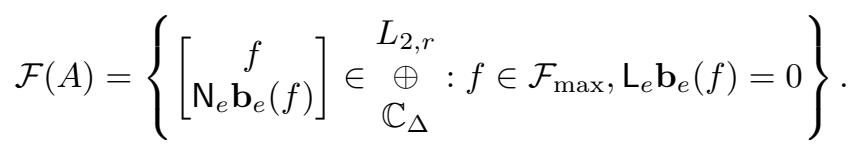

To construct an operator $W$ as in Theorem 2.2 we need to impose conditions on the coefficients $p$ and $r$ in (1.1). In all cases we need Condition 4.1 in a neighborhood of 0 , and in some cases we also need one of two Conditions, 5.1 or 5.2, on $r$ in neighborhoods of -1 or 1 . These will be discussed in Sections 4 and 5 respectively. 


\section{Smooth connection and associated operator}

To prepare the ground for the Conditions mentioned above (and in Part II), we develop the concept of smoothly connected half-neighbourhoods. A closed interval of non-zero length is said to be a left half-neighborhood of its right endpoint and a right half-neighborhood of its left endpoint.

Let $\imath$ be a closed subinterval of $[-1,1]$. By $\mathcal{F}_{\max }(\imath)$ we denote the set of all functions $f$ in $L_{2, r}(\imath)$ which are absolutely continuous on $\imath$ and such that $\int_{\imath} p\left|f^{\prime}\right|^{2}<$ $+\infty$. Note that $\mathcal{F}_{\max }[-1,1]$ is the space $\mathcal{F}_{\max }$ defined below Theorem 2.2 , In the next definition affine function $\alpha$ means $\alpha(t)=a+\alpha^{\prime} t$ where $a, \alpha^{\prime}, t \in \mathbb{R}$.

Definition 3.1. Let $p$ and $r$ be the coefficients in (1.1). Let $a, b \in[-1,1]$ and let $h_{a}$ and $h_{b}$, respectively, be half-neighborhoods of $a$ and $b$ which are contained in $[-1,1]$. We say that the ordered pair $\left(h_{a}, h_{b}\right)$ is smoothly connected if there exist

(a) positive real numbers $\epsilon$ and $\tau$,

(b) non-constant affine functions $\alpha:[0, \epsilon] \rightarrow h_{a}$ and $\beta:[0, \epsilon] \rightarrow h_{b}$,

(c) non-negative real functions $\rho$ and $\varpi$ defined on $[0, \epsilon]$,

such that

(i) $\alpha(0)=a$ and $\beta(0)=b$,

(ii) $p \circ \alpha$ and $p \circ \beta$ are locally integrable on the interval $(0, \epsilon]$,

(iii) $\rho \circ \alpha^{-1} \in \mathcal{F}_{\max }(\alpha([0, \epsilon]))$,

(iv) $1 / \tau<\varpi<\tau$ a.e. on $[0, \epsilon]$,

(v) $\quad \rho(t)=\frac{|r(\beta(t))|}{|r(\alpha(t))|}$, and $\varpi(t)=\frac{p(\beta(t))}{p(\alpha(t))}, \quad$ for $\quad t \in(0, \epsilon]$.

The numbers $\alpha^{\prime}, \beta^{\prime}$, (the slopes of $\alpha, \beta$, respectively) and $\rho(0)$ are called the parameters of the smooth connection.

Remark 3.2. Since the function $\alpha$ in Definition 3.1 is affine, the condition $\rho \circ \alpha^{-1} \in$ $\mathcal{F}_{\max }(\alpha([0, \epsilon]))$ in (iii) is equivalent to

$$
\rho \in A C[0, \epsilon] \quad \text { and } \quad \int_{0}^{\epsilon}\left|\rho^{\prime}(t)\right|^{2} p(\alpha(t)) d t<+\infty .
$$

Under the assumption that $1 / \tau<\varpi<\tau$ a.e. on $[0, \epsilon]$, it follows that property (3.1) is equivalent to

$$
\rho \in A C[0, \epsilon] \quad \text { and } \quad \int_{0}^{\epsilon}\left|\rho^{\prime}(t)\right|^{2} p(\beta(t)) d t<+\infty .
$$

To illustrate Definition 3.1, we make the following

Definition 3.3. Let $\nu$ and $a$ be real numbers and let $h_{a}$ be a half-neighborhood of $a$. Let $g$ be a function defined on $h_{a}$. Then $g$ is called of order $\nu$ on $h_{a}$ if there exists $g_{1} \in C^{1}\left(h_{a}\right)$ such that

$$
g(x)=(x-a)^{\nu} g_{1}(x) \quad \text { and } \quad g_{1}(x) \neq 0, \quad x \in h_{a} .
$$


Example 3.4. Let $a, b \in[-1,1]$. Let $h_{a}$ and $h_{b}$, respectively, be half-neighborhoods of $a$ and $b$ contained in $[-1,1]$. Assume that the coefficient $r$ in (1.1) is of order $\nu$ on both half-neighborhoods $h_{a}$ and $h_{b}$. Assume also that the functions $p$ and $1 / p$ are bounded on $h_{a}$ and $h_{b}$ (or, alternatively, that $p$ is of order $\mu$ on both halfneighborhoods $h_{a}$ and $h_{b}$.) Then lengthy, but straightforward, reasoning shows that the half-neighborhoods $h_{a}$ and $h_{b}$ are smoothly connected. Moreover the parameters of the smooth connection are nonzero numbers.

Remark 3.5. Throughout the paper we use the following convention: A product of functions is defined to have value 0 whenever one of its terms has value zero, even if some other terms are not defined.

Theorem 3.6. Let $\imath$ and $\jmath$ be closed intervals, $\imath, \jmath \in\{[-1,0],[0,1]\}$. Let $a$ be an endpoint of $\imath$ and let $b$ be an endpoint of $\jmath$. Denote by $a_{1}$ and $b_{1}$, respectively, the remaining endpoints. Assume that the half-neighborhoods $\imath$ of a and $\jmath$ of $b$ are smoothly connected with parameters $\alpha^{\prime}, \beta^{\prime}$ and $\rho(0)$. Then there exists an operator

$$
S: L_{2,|r|}(\imath) \rightarrow L_{2,|r|}(\jmath)
$$

such that:

$(S-1) \quad S \in \mathcal{L}\left(L_{2,|r|}(\imath), L_{2,|r|}(\jmath)\right), S^{*} \in \mathcal{L}\left(L_{2,|r|}(\jmath), L_{2,|r|}(\imath)\right)$.

$(S-2) \quad(S f)(x)=0,\left|x-b_{1}\right| \leq \frac{1}{2}$ for all $f \in L_{2,|r|}(\imath)$, and $\left(S^{*} g\right)(x)=0,\left|x-a_{1}\right| \leq \frac{1}{2}$ for all $g \in L_{2,|r|}(\jmath)$.

$(S-3) \quad S \mathcal{F}_{\max }(\imath) \subset \mathcal{F}_{\max }(\jmath), \quad S^{*} \mathcal{F}_{\max }(\jmath) \subset \mathcal{F}_{\max }(\imath)$.

$(S-4)$ For all $f \in \mathcal{F}_{\max }(\imath)$ and all $g \in \mathcal{F}_{\max }(\jmath)$ we have

$$
\lim _{\substack{y \rightarrow b \\ y \in J}}(S f)(y)=\left|\alpha^{\prime}\right| \lim _{\substack{x \rightarrow a \\ x \in \imath}} f(x), \quad \lim _{\substack{x \rightarrow a \\ x \in \imath}}\left(S^{*} g\right)(x)=\left|\beta^{\prime}\right| \rho(0) \lim _{\substack{y \rightarrow b \\ y \in J}} g(y) .
$$

Proof. Let $\epsilon>0$ be the real number and $\alpha$ and $\beta$ the affine functions introduced in Definition 3.1 Thus $\alpha(0)=a$ and $\beta(0)=b$. It is no loss of generality to assume that each of the intervals $\alpha([0, \epsilon])$ and $\beta([0, \epsilon])$ has a length $<1 / 2$. Let $\alpha_{1}:[0,1] \rightarrow \imath$ and $\beta_{1}:[0,1] \rightarrow \jmath$ be strictly monotonic and continuously differentiable bijections such that $\alpha_{1}(x)=\alpha(x)$ and $\beta_{1}(x)=\beta(x)$ for all $x \in[0, \epsilon]$. Then $\alpha_{1}(1)=a_{1}$ and $\beta_{1}(1)=b_{1}$.

Let $\phi:[0,1] \rightarrow[0,1], \phi \in C^{1}[0,1]$, be such that

$$
\phi(t)=1, \quad 0 \leq t \leq \epsilon / 2, \quad \phi(t)=0, \quad \epsilon \leq t \leq 1 .
$$

Define the operator $S: L_{2,|r|}(\imath) \rightarrow L_{2,|r|}(\jmath)$ by

$$
(S f)\left(\beta_{1}(t)\right):=\left|\alpha^{\prime}\right| f\left(\alpha_{1}(t)\right) \phi(t), \quad f \in L_{2,|r|}(\imath), \quad t \in[0,1] .
$$

Clearly $S$ is linear.

In what follows we shall use the combination of property (3.2) and Remark 3.5 to simplify the notation and calculations. For example these imply that in the definition (3.3) of $S$ we could use $\beta$ and $\alpha$ instead of $\beta_{1}$ and $\alpha_{1}$ without changing the substance of the definition. 
At various points of the proof we shall employ the monotonic (increasing or decreasing) substitutions

$$
x=\beta(t), \quad \alpha(t)=\xi .
$$

To prove that $S$ is bounded we let $f \in L_{2,|r|}(\imath)$ and calculate

$$
\begin{aligned}
\int_{\jmath}|(S f)(x)|^{2}|r(x)| d x & =\operatorname{sgn}\left(\beta^{\prime}\right) \int_{0}^{\epsilon}|(S f)(\beta(t))|^{2}|r(\beta(t))| \beta^{\prime} d t \\
& =\left|\alpha^{\prime}\right|^{2}\left|\beta^{\prime}\right| \int_{0}^{\epsilon}|f(\alpha(t))|^{2}|\phi(t)|^{2} \rho(t)|r(\alpha(t))| d t \\
& \leq\left|\alpha^{\prime}\right|^{2}\left|\beta^{\prime}\right| R \int_{0}^{\epsilon}|f(\alpha(t))|^{2}|\phi(t)|^{2}|r(\alpha(t))| d x \\
& \leq\left|\alpha^{\prime}\right|\left|\beta^{\prime}\right| R \int_{\imath}|f(\xi)|^{2}|r(\xi)| d \xi
\end{aligned}
$$

where $R$ is an upper bound of the function $\rho$. The above calculation proves that $S$ is bounded and $\|S\| \leq\left|\alpha^{\prime}\right|\left|\beta^{\prime}\right| R$.

To verify the first claim in $(S-21)$, let $\left|x-b_{1}\right|<1 / 2$ and $f \in L_{2,|r|}(\imath)$. Note that the length of $\jmath$ is 1 , the endpoints of $\jmath$ are $b, b_{1}$, and $\beta(0)=b$. Since $\beta([0, \epsilon])$ has the length $<1 / 2$ and since $\beta_{1}$ is strictly monotonic we conclude that $t=\beta_{1}^{-1}(x)>\epsilon$. Therefore, by (3.2),

$$
(S f)(x)=\left|\alpha^{\prime}\right| f(\alpha(t)) \phi(t)=0 .
$$

This proves the first claim in $(S-2)$.

To prove $S \mathcal{F}_{\max }(\imath) \subset \mathcal{F}_{\max }(\jmath)$, let $f \in \mathcal{F}_{\max }(\imath)$. By definition (3.3), since $f$ is absolutely continuous on $\imath$ and $\phi \in C^{1}[0,1]$, the function $S f$ is absolutely continuous on $\jmath$ and for almost all $t \in[0,1]$ we have

$$
\beta^{\prime}(S f)^{\prime}(\beta(t))=\left|\alpha^{\prime}\right|\left(\alpha^{\prime} f^{\prime}(\alpha(t)) \phi(t)+f(\alpha(t)) \phi^{\prime}(t)\right) .
$$

To prove that $S f \in \mathcal{F}_{\max }(\jmath)$ we need to show that $(S f)^{\prime} \in L_{2, p}(\jmath)$, that is

$$
\int_{\jmath}\left|(S f)^{\prime}(x)\right|^{2} p(x) d x=\left|\beta^{\prime}\right| \int_{0}^{\epsilon}\left|(S f)^{\prime}(\beta(t))\right|^{2} p(\beta(t)) d t<+\infty .
$$

We consider each summand in (3.4) separately. By (3.2), the second function in the sum in (3.4) is a continuous function which vanishes outside of the interval $[\epsilon / 2, \epsilon]$. Since by assumption $p \circ \beta$ is an integrable function on $[\epsilon / 2, \epsilon]$, it follows that

$$
\int_{0}^{1}\left|f(\alpha(t)) \phi^{\prime}(t)\right|^{2} p(\beta(t)) d t<+\infty
$$


Using the notation and assumptions from Definition 3.1, for the first function in the sum in (3.4) we have

$$
\begin{aligned}
\int_{0}^{1}\left|f^{\prime}(\alpha(t))\right|^{2}|\phi(t)|^{2} p(\beta(t)) d t & =\int_{0}^{\epsilon}\left|f^{\prime}(\alpha(t))\right|^{2}|\phi(t)|^{2} \varpi(t) p(\alpha(t)) d t \\
& \leq \tau \int_{0}^{\epsilon}\left|f^{\prime}(\alpha(t))\right|^{2}|\phi(t)|^{2} p(\alpha(t)) d t \\
& \leq \frac{\tau}{\left|\alpha^{\prime}\right|} \int_{\imath}\left|f^{\prime}(\xi)\right|^{2} p(\xi) d \xi
\end{aligned}
$$

Since $f^{\prime} \in L_{2, p}(\imath)$ the last expression is finite. Based on (3.4), (3.5), (3.6) and (3.7) we conclude that $(S f)^{\prime} \in L_{2, p}(\jmath)$ and consequently $S f \in \mathcal{F}_{\max }(\jmath)$.

The next step in the proof is to calculate

$$
S^{*}: L_{2,|r|}(\jmath) \rightarrow L_{2,|r|}(\imath) .
$$

Note that $S^{*}$ is calculated with respect to the Hilbert space inner products on the underlying spaces. Property (3.2) allows us to consider only affine changes of variable in the integrals below. Let $f \in L_{2,|r|}(\imath)$ and $g \in L_{2,|r|}(\jmath)$. Then

$$
\begin{aligned}
\int_{\jmath}(S f)(x) \overline{g(x)}|r(x)| d x \\
\quad=\left|\beta^{\prime}\right| \int_{0}^{\epsilon}(S f)(\beta(t)) \overline{g(\beta(t))}|r(\beta(t))| d t \\
=\left|\beta^{\prime}\right|\left|\alpha^{\prime}\right| \int_{0}^{\epsilon} f(\alpha(t)) \phi(t) \overline{g(\beta(t))} \rho(t)|r(\alpha(t))| d t \\
=\left|\beta^{\prime}\right| \int_{\imath} f(\xi) \phi\left(\alpha^{-1}(\xi)\right) \overline{g\left(\beta\left(\alpha^{-1}(\xi)\right)\right)} \rho\left(\alpha^{-1}(\xi)\right)|r(\xi)| d \xi
\end{aligned}
$$

Therefore for $g \in L_{2,|r|}(\jmath)$ we have

$$
\left(S^{*} g\right)(x):=\left|\beta^{\prime}\right|(\rho \phi(g \circ \beta))\left(\alpha^{-1}(x)\right), \quad x \in \imath .
$$

Thus

$$
\left(S^{*} g\right)(\alpha(t))=\left|\beta^{\prime}\right| g(\beta(t)) \rho(t) \phi(t), \quad g \in L_{2,|r|}(\jmath), \quad t \in[0,1] .
$$

As the adjoint of a bounded operator, the operator $S^{*}$ is bounded. To verify the second part of $(S-2)$ let $\left|x-a_{1}\right|<1 / 2$ and $g \in L_{2,|r|}(\jmath)$. Note that the length of $\imath$ is 1 and $\alpha(0)=a$. Since $\alpha([0, \epsilon])$ has length $<1 / 2$ and since $\alpha_{1}$ is strictly monotonic we conclude that $t=\alpha_{1}^{-1}(x)>\epsilon$. Therefore, by (3.2),

$$
\left(S^{*} g\right)(x)=\left|\beta^{\prime}\right| g(\beta(t)) \rho(t) \phi(t)=0 \text {. }
$$

To prove $S^{*} \mathcal{F}_{\max }(\jmath) \subset \mathcal{F}_{\max }(\imath)$, let $g \in \mathcal{F}_{\max }(\jmath)$. Since $g$ and $\rho$ are absolutely continuous and $\phi \in C^{1}[0,1]$, the function $\left(S^{*} g\right) \circ \alpha$ is absolutely continuous on $[0,1]$. Differentiation of (3.8) yields

$$
\begin{aligned}
& \alpha^{\prime}\left(S^{*} g\right)^{\prime}(\alpha(t)) \\
& \quad=\left|\beta^{\prime}\right|\left(\left(\rho^{\prime} \phi(g \circ \beta)\right)(t)+\left(\rho \phi^{\prime}(g \circ \beta)\right)(t)+\beta^{\prime}\left(\rho \phi\left(g^{\prime} \circ \beta\right)\right)(t)\right),
\end{aligned}
$$


for almost all $t \in[0,1]$. To prove that $S^{*} f \in \mathcal{F}_{\max }(\imath)$ we need to show that $\left(S^{*} f\right)^{\prime} \in L_{2, p}(\imath)$, that is

$$
\int_{\imath}\left|\left(S^{*} f\right)^{\prime}(\xi)\right|^{2} p(\xi) d \xi=\left|\alpha^{\prime}\right| \int_{0}^{\epsilon}\left|\left(S^{*} f\right)^{\prime}(\alpha(t))\right|^{2} p(\alpha(t)) d t<+\infty .
$$

We prove that each summand on the right-hand side of (3.9) belongs to $L_{2, p}(\imath)$.

By (3.2), the second summand is a continuous function which vanishes outside of the interval $[\epsilon / 2, \epsilon]$. Since $p \circ \alpha$ is an integrable function on $[\epsilon / 2, \epsilon]$, it follows that

$$
\int_{0}^{1}\left|g(\beta(t)) \phi^{\prime}(t) \rho(t)\right|^{2} p(\alpha(t)) d t<+\infty .
$$

Next, we consider the third summand in (3.9). Since $\rho$ is continuous on $[0,1]$ we can consider only $\phi\left(g^{\prime} \circ \beta\right)$ :

$$
\begin{aligned}
\int_{0}^{1}\left|g^{\prime}(\beta(t)) \phi(t)\right|^{2} p(\alpha(t)) d t & =\int_{0}^{\epsilon}\left|g^{\prime}(\beta(t)) \phi(t)\right|^{2} \frac{1}{\varpi(t)} p(\beta(t)) d t \\
& \leq \tau \int_{0}^{\epsilon}\left|g^{\prime}(\beta(t)) \phi(t)\right|^{2} p(\beta(t)) d t \\
& =\frac{\tau}{\left|\beta^{\prime}\right|} \int_{\jmath}\left|g^{\prime}(x)\right|^{2} p(x) d x<+\infty
\end{aligned}
$$

Finally, for the first summand in (3.9), it is sufficient to consider $\rho^{\prime} \phi$, since $g \circ \beta$ is absolutely continuous. By (3.1)

$$
\int_{0}^{1}\left|\rho^{\prime}(t) \phi(t)\right|^{2} p(\alpha(t)) d t \leq \int_{0}^{\epsilon}\left|\rho^{\prime}(t)\right|^{2} p(\alpha(t)) d t<+\infty .
$$

Based on (3.9), (3.10), (3.11), (3.12) and (3.13) we conclude that $\left(S^{*} f\right)^{\prime} \in L_{2, p}(\imath)$ and consequently $S^{*} f \in \mathcal{F}_{\max }(\imath)$.

Thus we have verified the properties $(S-\mathbb{1}),(S-2),(S-3)$. Since $(S-4)$ is clear the theorem is proved.

\section{Condition at 0 and associated operator}

Condition 4.1 (Condition at 0 ). Let $p$ and $r$ be coefficients in (1.1). Denote by $0_{-}$ a generic left and by $0_{+}$a generic right half-neighborhood of 0 . We assume that at least one of the four ordered pairs of half-neighborhoods

$$
\left(0_{-}, 0_{-}\right), \quad\left(0_{-}, 0_{+}\right), \quad\left(0_{+}, 0_{-}\right), \quad\left(0_{+}, 0_{+}\right),
$$

is smoothly connected with connection parameters $\alpha_{0}^{\prime}, \beta_{0}^{\prime}$ and $\rho_{0}(0)$ such that $\left|\alpha_{0}^{\prime}\right| \neq\left|\beta_{0}^{\prime}\right| \rho_{0}(0)$.

Theorem 4.2. Assume that the coefficients $p$ and $r$ satisfy Condition 4.1. Then there exists an operator $W_{0}: L_{2, r} \rightarrow L_{2, r}$ such that

(a) $W_{0}$ is bounded on $L_{2,|r|}$. 
(b) The operator $J_{0} W_{0}-I$ is nonnegative on the Hilbert space $L_{2,|r|}$. In particular $W_{0}^{-1}$ is bounded and $W_{0}$ is positive on the Krein space $L_{2, r}$.

(c) $\left(W_{0} f\right)(x)=(J f)(x), \quad \frac{1}{2} \leq|x| \leq 1, \quad f \in L_{2, r}$.

(d) $W_{0} \mathcal{F}_{\max }[-1,1] \subset \mathcal{F}_{\max }[-1,1]$.

Proof. Let $\alpha_{0}^{\prime}, \beta_{0}^{\prime}$, and $\rho_{0}(0)$ be given by Condition 4.1 Recall that $\left|\alpha_{0}^{\prime}\right| \neq\left|\beta_{0}^{\prime}\right| \rho_{0}(0)$. Let $\phi_{0}:[-1,1] \rightarrow[0,1], \phi_{0} \in C^{1}[-1,1]$ be an even function such that

$$
\phi_{0}(0)=1 \quad \text { and } \quad \phi_{0}(x)=0 \quad \text { for } \quad 1 / 2 \leq|x| \leq 1 .
$$

Define the operators

$$
P_{0,-}: L_{2,|r|}(-1,0) \rightarrow L_{2,|r|}(-1,0) \quad \text { and } \quad P_{0,+}: L_{2,|r|}(0,1) \rightarrow L_{2,|r|}(0,1)
$$

by

$$
\begin{array}{lll}
\left(P_{0,-} f\right)(x)=f(x) \phi_{0}(x), & f \in L_{2,|r|}(-1,0), & x \in[-1,0], \\
\left(P_{0,+} f\right)(x)=f(x) \phi_{0}(x), & f \in L_{2,|r|}(0,1), & x \in[0,1] .
\end{array}
$$

Then $P_{0,-}$ and $P_{0,+}$ are self-adjoint operators with the following properties:

$$
\begin{aligned}
\left(P_{0,-} f\right)(x) & =0, \quad f \in L_{2,|r|}(-1,0), \quad-1 \leq x \leq-\frac{1}{2}, \\
\left(P_{0,+} f\right)(x) & =0, \quad f \in L_{2,|r|}(0,1), \quad \frac{1}{2} \leq x \leq 1, \\
P_{0,-} \mathcal{F}_{\max }[-1,0] & \subset \mathcal{F}_{\max }[-1,0], \quad P_{0,+} \mathcal{F}_{\max }[0,1] \subset \mathcal{F}_{\max }[0,1],
\end{aligned}
$$

and

$$
\begin{array}{ll}
\left(P_{0,-} f\right)(0-)=f(0-), & f \in \mathcal{F}_{\max }[-1,0], \\
\left(P_{0,+} f\right)(0+)=f(0+), & f \in \mathcal{F}_{\max }[0,1] .
\end{array}
$$

Here, the value of a function at $0 \pm$ represents its one sided limit.

Condition 4.1 1requires that one of the four ordered pairs of half neighborhoods is smoothly connected. For such a pair, Theorem 3.6 guarantees the existence of a specific operator which we denote by $S_{0}$. For each of the four pairs we shall use different combinations of scaled operators $P_{0,-}, P_{0,+}, S_{0}$ and $S_{0}^{*}$ to define a bounded block operator

$$
X_{0}: \begin{gathered}
L_{2,|r|}(-1,0) \\
\oplus \\
L_{2,|r|}(0,1)
\end{gathered} \rightarrow \begin{gathered}
L_{2,|r|}(-1,0) \\
L_{2,|r|}(0,1)
\end{gathered}
$$

with the following properties

$$
\begin{aligned}
\left(X_{0}^{*} f\right)(x) & =0, \quad 1 / 2 \leq|x| \leq 1, \\
X_{0} \mathcal{F}_{\max }[-1,1] & \subset \mathcal{F}_{\max }[-1,1], \\
\left(X_{0} f\right)(0) & =f(0), \quad f \in \mathcal{F}_{\max }[-1,1], \\
X_{0}^{*} \mathcal{F}_{\max }[-1,1] & \subset \mathcal{F}_{\max }[-1,0] \oplus \mathcal{F}_{\max }[0,1], \\
\left(X_{0}^{*} f\right)(0+)+\left(X_{0}^{*} f\right)(0-) & =-2 f(0), \quad f \in \mathcal{F}_{\max }[-1,1] .
\end{aligned}
$$


These properties of $X_{0}$ and $X_{0}^{*}$ imply that the operator

$$
W_{0}=J\left(X_{0}^{*} X_{0}+I\right)
$$

has all the properties stated in the theorem.

Since we assume that $\left|\alpha_{0}^{\prime}\right| \neq\left|\beta_{0}^{\prime}\right| \rho_{0}(0)$, the system

$$
\gamma_{1}\left|\alpha_{0}^{\prime}\right|+\gamma_{2}=1, \quad \gamma_{1}\left|\beta_{0}^{\prime}\right| \rho_{0}(0)+\gamma_{2}=-3
$$

has a nontrivial real solution $\gamma_{1}, \gamma_{2}$. We use this solution in the definitions below.

Case 1. Assume that the half-neighborhoods $0_{-}, 0_{-}$in (4.1) are smoothly connected. Then by Theorem 3.6 there exists an operator

$$
S_{0}: L_{2,|r|}(-1,0) \rightarrow L_{2,|r|}(-1,0)
$$

which satisfies $(S-1)-(S-4)$ in Theorem 3.6 with $\imath=\jmath=[-1,0], a=b=0$. In particular, for $f \in \mathcal{F}_{\max }[-1,0]$,

$$
\left(S_{0} f\right)(0-)=\left|\alpha_{0}^{\prime}\right| f(0-), \quad\left(S_{0}^{*} f\right)(0-)=\left|\beta_{0}^{\prime}\right| \rho_{0}(0) f(0-) .
$$

We define $X_{0}$ and calculate $X_{0}^{*}$ as

$$
X_{0}=\left[\begin{array}{cc}
\gamma_{1} S_{0}+\gamma_{2} P_{0,-} & 0 \\
0 & P_{0,+}
\end{array}\right], \quad X_{0}^{*}=\left[\begin{array}{cc}
\gamma_{1} S_{0}^{*}+\gamma_{2} P_{0,-} & 0 \\
0 & P_{0,+}
\end{array}\right]
$$

Case 2. Assume that the half-neighborhoods $0_{-}, 0_{+}$in (4.1) are smoothly connected. Then by Theorem 3.6 there exists an operator

$$
S_{0}: L_{2,|r|}(-1,0) \rightarrow L_{2,|r|}(0,1)
$$

which satisfies $(S-1)-(S-4)$ in Theorem 3.6 with $\imath=[-1,0], \jmath=[0,1], a=b=0$. In particular, for $f \in \mathcal{F}_{\max }[-1,0]$,

$$
\left(S_{0} f\right)(0+)=\left|\alpha_{0}^{\prime}\right| f(0-), \quad\left(S_{0}^{*} f\right)(0-)=\left|\beta_{0}^{\prime}\right| \rho_{0}(0) f(0+) .
$$

We define $X_{0}$ and calculate $X_{0}^{*}$ as

$$
X_{0}=\left[\begin{array}{cc}
P_{0,-} & 0 \\
\gamma_{1} S_{0} & \gamma_{2} P_{0,+}
\end{array}\right], \quad X_{0}^{*}=\left[\begin{array}{cc}
P_{0,-} & \gamma_{1} S_{0}^{*} \\
0 & \gamma_{2} P_{0,+}
\end{array}\right] .
$$

Case 3. Assume that the half-neighborhoods $0_{+}, 0_{-}$in (4.1) are smoothly connected. Then by Theorem 3.6 there exists an operator

$$
S_{0}: L_{2,|r|}(0,1) \rightarrow L_{2,|r|}(-1,0)
$$

which satisfies $(S-1)-(S-4)$ in Theorem 3.6 with $\imath=[0,1], \jmath=[-1,0], a=b=0$. In particular, for $f \in \mathcal{F}_{\max }[0,1]$,

$$
\left(S_{0} f\right)(0-)=\left|\alpha_{0}^{\prime}\right| f(0+), \quad\left(S_{0}^{*} f\right)(0+)=\left|\beta_{0}^{\prime}\right| \rho_{0}(0) f(0-) .
$$

We define $X_{0}$ and calculate $X_{0}^{*}$ as

$$
X_{0}=\left[\begin{array}{cc}
\gamma_{2} P_{0,-} & \gamma_{1} S_{0} \\
0 & P_{0,+}
\end{array}\right], \quad X_{0}^{*}=\left[\begin{array}{cc}
\gamma_{2} P_{0,-} & 0 \\
\gamma_{1} S_{0}^{*} & P_{0,+}
\end{array}\right]
$$


Case 4. Assume that the half-neighborhoods $0_{+}, 0_{+}$in (4.1) are smoothly connected. Then by Theorem 3.6 there exists an operator

$$
S_{0}: L_{2,|r|}(0,1) \rightarrow L_{2,|r|}(0,1)
$$

which satisfies $(S-1)-(S-4)$ in Theorem 3.6 with $\imath=[0,1], \jmath=[0,1], a=b=0$. In particular, for $f \in \mathcal{F}_{\max }[0,1]$,

$$
\left(S_{0} f\right)(0+)=\left|\alpha_{0}^{\prime}\right| f(0+), \quad\left(S_{0}^{*} f\right)(0+)=\left|\beta_{0}^{\prime}\right| \rho_{0}(0) f(0+) .
$$

We define $X_{0}$ and calculate $X_{0}^{*}$ as

$$
X_{0}=\left[\begin{array}{cc}
P_{0,-} & 0 \\
0 & \gamma_{1} S_{0}+\gamma_{2} P_{0,+}
\end{array}\right], \quad X_{0}^{*}=\left[\begin{array}{cc}
P_{0,-} & 0 \\
0 & \gamma_{1} S_{0}^{*}+\gamma_{2} P_{0,+}
\end{array}\right] .
$$

First note that in each of the four cases above, the operator $X_{0}$ is bounded since each of its components is bounded.

In each of the four cases above, the property (4.7) follows from $(S-2)$ in Theorem 3.6. and properties (4.2) and (4.3).

Let $f \in \mathcal{F}_{\max }[-1,1]$. Since $\gamma_{1}\left|\alpha_{0}^{\prime}\right|+\gamma_{2}=1$, the function $X_{0} f$ is continuous in each case and $\left(X_{0} f\right)(0)=f(0)$. This, (4.4), (4.5), (4.6), $(S-3)$ and $(S$-4) in Theorem 3.6 imply (4.8). Inclusion (4.10) follows similarly.

In each of the above cases, equation (4.11) is a consequence of

$$
\gamma_{1}\left|\beta_{0}^{\prime}\right| \rho_{0}(0)+\gamma_{2}=-3,
$$

(4.5), (4.6) and $(S-4)$ in Theorem 3.6. This proves the theorem.

Remark 4.3. Note the behavior of the operator $W_{0}$ in Theorem 4.2 at the boundary of the interval $[-1,1]$ :

$$
\left[\begin{array}{c}
\left(W_{0} f\right)(-1) \\
\left(W_{0} f\right)(1)
\end{array}\right]=\left[\begin{array}{c}
-f(-1) \\
f(1)
\end{array}\right], \quad f \in \mathcal{F}_{\max }[-1,1] .
$$

This property of $W_{0}$ will be used in Section 6 . In the next section, under additional assumptions on the coefficients $p$ and $r$ in a neighborhood of -1 and 1 , we shall construct operators $W$ with specified behaviors at -1 and 1 .

\section{Conditions at $\mathbf{- 1}$ and $\mathbf{1}$, and associated operators}

In this section we show that under additional assumptions on the coefficients $p$ and $r$ near -1 we can construct an operator $W_{-1}$ with prescribed behavior at -1 and under additional assumptions near 1 we can construct an operator $W_{+1}$ with prescribed behavior at 1 .

Condition 5.1 (Condition at -1). Let $p$ and $r$ be coefficients in (1.1). We assume that a right half-neighborhood of -1 is smoothly connected to a right half-neighborhood of -1 with the connection parameters $\alpha_{-1}^{\prime}, \beta_{-1}^{\prime}$ and $\rho_{-1}(0)$ such that $\left|\alpha_{-1}^{\prime}\right| \neq\left|\beta_{-1}^{\prime}\right| \rho_{-1}(0)$. 
Condition 5.2 (Condition at 1). Let $p$ and $r$ be coefficients in (1.1). We assume that a left half-neighborhood of 1 is smoothly connected to a left halfneighborhood of 1 with the connection parameters $\alpha_{+1}^{\prime}, \beta_{+1}^{\prime}$ and $\rho_{+1}(0)$ such that $\left|\alpha_{+1}^{\prime}\right| \neq\left|\beta_{+1}^{\prime}\right| \rho_{+1}(0)$.

In the rest of this section we shall need two operators analogous to $P_{0,-}$ and $P_{0,+}$ introduced in Section 4. Let $\phi_{1}:[-1,1] \rightarrow[0,1]$ be a smooth even function such that

$$
\phi_{1}(-1)=1, \quad \phi_{1}(x)=0 \quad \text { for } \quad 0 \leq|x| \leq 1 / 2, \quad \phi_{1}(1)=1 .
$$

Define the operators

$$
P_{1,-}: L_{2,|r|}(-1,0) \rightarrow L_{2,|r|}(-1,0) \quad \text { and } \quad P_{1,+}: L_{2,|r|}(0,1) \rightarrow L_{2,|r|}(0,1)
$$

by

$$
\begin{aligned}
& \left(P_{1,-} f\right)(x)=f(x) \phi_{1}(x), \quad f \in L_{2,|r|}(-1,0), \quad x \in[-1,0], \\
& \left(P_{1,+} f\right)(x)=f(x) \phi_{1}(x), \quad f \in L_{2,|r|}(0,1), \quad x \in[0,1] \text {. }
\end{aligned}
$$

Then $P_{1,-}$ and $P_{1,+}$ are self-adjoint operators with the following properties:

$$
\begin{aligned}
\left(P_{1,-} f\right)(x) & =0, \quad f \in L_{2,|r|}(-1,0), \quad-\frac{1}{2} \leq x \leq 0, \\
\left(P_{1,+} f\right)(x) & =0, \quad f \in L_{2,|r|}(0,1), \quad 0 \leq x \leq \frac{1}{2}, \\
P_{1,-} \mathcal{F}_{\max }[-1,0] & \subset \mathcal{F}_{\max }[-1,0], \quad P_{1,+} \mathcal{F}_{\max }[0,1] \subset \mathcal{F}_{\max }[0,1],
\end{aligned}
$$

and

$$
\begin{aligned}
\left(P_{1,-} f\right)(-1+) & =f(-1+), \quad f \in \mathcal{F}_{\max }[-1,0], \\
\left(P_{1,+} f\right)(1-) & =f(1-), \quad f \in \mathcal{F}_{\max }[0,1] .
\end{aligned}
$$

Proposition 5.3. Assume that the coefficients $p$ and $r$ satisfy Condition [5.1. Let $\mu$ be an arbitrary complex number. Then there exists an operator $W_{-1}: L_{2, r} \rightarrow L_{2, r}$ such that

(a) $W_{-1}$ is bounded on $L_{2,|r|}$.

(b) The operator $J_{0} W_{-1}-I$ is nonnegative on the Hilbert space $L_{2,|r|}$. In particular $\left(W_{-1}\right)^{-1}$ is bounded and $W_{-1}$ is positive on the Krein space $L_{2, r}$.

(c) $\left(W_{-1} f\right)(x)=(J f)(x), \quad-\frac{1}{2} \leq x \leq 1, \quad f \in L_{2, r}$.

(d) $W_{-1} \mathcal{F}_{\max }[-1,1] \subset \mathcal{F}_{\max }[-1,0] \oplus \mathcal{F}_{\max }[0,1]$.

(e) $\left(W_{-1} f\right)(-1)=\mu f(-1)$ for all $f \in \mathcal{F}_{\max }[-1,1]$.

Proof. We use the notation introduced in Condition 5.1. By Theorem 3.6 there exists a bounded operator $S_{-1}: L_{2,|r|}(-1,0) \rightarrow L_{2,|r|}(-1,0)$ such that

$$
S_{-1} \mathcal{F}_{\max }[-1,0] \subset \mathcal{F}_{\max }[-1,0] \text { and } S_{-1}^{*} \mathcal{F}_{\max }[-1,0] \subset \mathcal{F}_{\max }[-1,0] \text {, }
$$

and, for all $f \in \mathcal{F}_{\max }[-1,0]$,

$$
\left(S_{-1} f\right)(-1)=\left|\alpha_{-1}^{\prime}\right| f(-1), \quad\left(S_{-1}^{*} f\right)(-1)=\left|\beta_{-1}^{\prime}\right| \rho_{-1}(0) f(-1) .
$$


Let $\mu$ be an arbitrary complex number. Since we assume that $\left|\alpha_{-1}^{\prime}\right| \neq\left|\beta_{-1}^{\prime}\right| \rho_{-1}(0)$, the complex numbers $\gamma_{1}$ and $\gamma_{2}$ can be chosen such that

$$
\gamma_{1}\left|\alpha_{-1}^{\prime}\right|+\gamma_{2}=1, \quad \bar{\gamma}_{1}\left|\beta_{-1}^{\prime}\right| \rho_{-1}(0)+\bar{\gamma}_{2}=-\mu-1
$$

We define $X_{-1}$ and calculate $X_{-1}^{*}$ as

$$
X_{-1}=\left[\begin{array}{cc}
\gamma_{1} S_{-1}+\gamma_{2} P_{1,-} & 0 \\
0 & 0
\end{array}\right], \quad X_{-1}^{*}=\left[\begin{array}{cc}
\bar{\gamma}_{1} S_{-1}^{*}+\bar{\gamma}_{2} P_{1,-} & 0 \\
0 & 0
\end{array}\right]
$$

Then for all $f \in \mathcal{F}_{\max }[-1,1]$ we have

$$
\left(X_{-1} f\right)(-1)=f(-1) \text { and } \quad\left(X_{-1}^{*} f\right)(-1)=(-\mu-1) f(-1) .
$$

Therefore

$$
W_{-1}=J\left(X_{-1}^{*} X_{-1}+I\right)
$$

has all the properties stated in the proposition.

The proof of the next proposition is very similar to the preceding proof, and will be omitted.

Proposition 5.4. Assume that the coefficients $p$ and $r$ satisfy Condition 5.2, Let $\mu$ be an arbitrary complex number. Then there exists an operator

$$
W_{+1}: L_{2, r} \rightarrow L_{2, r}
$$

such that

(a) $W_{+1}$ is bounded on $L_{2,|r|}$.

(b) The operator $J_{0} W_{+1}-I$ is nonnegative on the Hilbert space $L_{2,|r|}$. In particular $\left(W_{+1}\right)^{-1}$ is bounded and $W_{+1}$ is positive on the Krein space $L_{2, r}$.

(c) $\left(W_{+1} f\right)(x)=(J f)(x), \quad-1 \leq x \leq \frac{1}{2}, \quad f \in L_{2, r}$.

(d) $W_{+1} \mathcal{F}_{\max }[-1,1] \subset \mathcal{F}_{\max }[-1,0] \oplus \mathcal{F}_{\max }[0,1]$.

(e) $\left(W_{+1} f\right)(1)=\mu f(1)$ for all $f \in \mathcal{F}_{\max }[-1,1]$.

\section{Riesz basis of root vectors}

In this section we return to the eigenvalue problem (1.1)-(1.2) and the operator $A$ associated with it. We start with cases when the conditions in Section 5 are not needed. We remark that the notation of Section 2 is used extensively in the rest of this section.

Theorem 6.1. Assume that the following three conditions are satisfied.

(a) The coefficients $p$ and $r$ satisfy Condition 4.1.

(b) One of the following is true:

(i) $\mathrm{L}_{n} \neq 0$,

(ii) $\mathrm{L}=\left[\begin{array}{llll}1 & 0 & 0 & 0\end{array}\right]$,

(iii) $\mathrm{L}=\left[\begin{array}{llll}0 & 1 & 0 & 0\end{array}\right]$.

(c) One of the following is true:

(i) $\mathrm{N}_{n} \neq 0$, 
(ii) $\mathrm{N}=\left[\begin{array}{llll}1 & 0 & 0 & 0\end{array}\right]$ and $\Delta<0$,

(iii) $\mathrm{N}=\left[\begin{array}{llll}0 & 1 & 0 & 0\end{array}\right]$ and $\Delta>0$.

Then there is a basis for each root subspace of $A$, so that the union of all these bases is a Riesz basis of $L_{2,|r|} \oplus \mathbb{C}_{|\Delta|}$.

Proof. Assume first that $\mathrm{N}_{n} \neq 0$. By (2.7), the form domain of $A$ when $\mathrm{L}_{n} \neq 0$ is given by

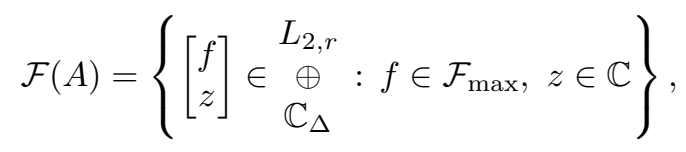

and in the other two cases in (b), (2.8) gives

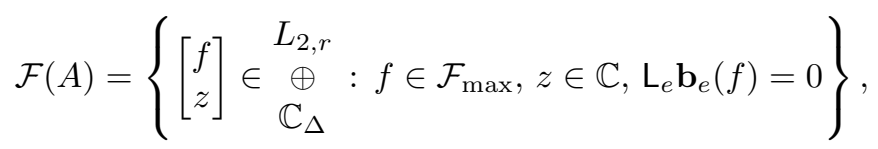

where $\mathbf{L}_{e} \mathbf{b}_{e}(f)=f(-1)$ in case (b-ii) and $\mathbf{L}_{e} \mathbf{b}_{e}(f)=f(1)$ in case (b-iii).

Next assume ([C-ii). Then $\mathrm{N}_{e} \mathbf{b}_{e}(f)=f(-1)$ and (2.9) shows that

$$
\mathcal{F}(A)=\left\{\left[\begin{array}{c}
f \\
f(-1)
\end{array}\right] \in \begin{array}{c}
L_{2, r} \\
\underset{\mathbb{C}_{\Delta}}{\oplus}: f \in \mathcal{F}_{\max }
\end{array}\right\}
$$

when $\mathrm{L}_{n} \neq 0$, and in the other cases in (b), (2.10) gives

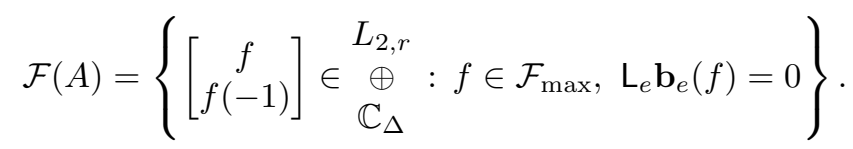

Finally, assume (criii). Then $\mathrm{N}_{e} \mathbf{b}_{e}(f)=f(1)$ and (2.9) shows that

$$
\mathcal{F}(A)=\left\{\left[\begin{array}{c}
f \\
f(1)
\end{array}\right] \in \begin{array}{c}
L_{2, r} \\
\underset{\mathbb{C}_{\Delta}}{\oplus}: f \in \mathcal{F}_{\max }
\end{array}\right\}
$$

when $\mathrm{L}_{n} \neq 0$, and in the other cases in (b), (2.10) gives

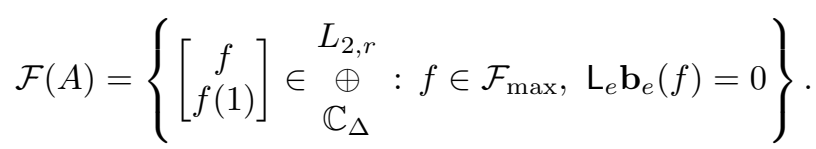

Let $W_{0}$ be the operator constructed in Theorem 4.2, and let

$$
W=\left[\begin{array}{cc}
W_{0} & 0 \\
0 & \operatorname{sgn}(\Delta)
\end{array}\right]: \begin{array}{ccc}
L_{2, r} & & L_{2, r} \\
\mathbb{C}_{\Delta} & \rightarrow & \oplus \\
\mathbb{C}_{\Delta}
\end{array}
$$

A straightforward verification shows that $W$ is a bounded, boundedly invertible, positive operator in the Krein space $L_{2, r} \oplus \mathbb{C}_{\Delta}$ and $W \mathcal{F}(A) \subset \mathcal{F}(A)$ in each of the above listed cases. Consequently, the theorem follows from Theorem 2.2 
In the next result we shall assume that one of the conditions from Section 5 is satisfied.

Theorem 6.2. Assume that the following three conditions are satisfied.

(a) The coefficients $p$ and $r$ satisfy Condition 4.1, and one of Conditions 5.1, 5.2.

(b) $\mathrm{N}_{n} \neq 0$.

(c) $\mathrm{L}=\left[\begin{array}{llll}u & v & 0 & 0\end{array}\right]$ with $u v \neq 0$.

Then there is a basis for each root subspace of $A$, so that the union of all these bases is a Riesz basis of $L_{2,|r|} \oplus \mathbb{C}_{|\Delta|}$.

Proof. Under the assumptions of the theorem, (2.8) shows that the form domain of $A$ is given by

$$
\mathcal{F}(A)=\left\{\left[\begin{array}{l}
f \\
z
\end{array}\right] \underset{\substack{\mathbb{C}_{\Delta}}}{\underset{L_{2, r}}{\in}}: f \in \mathcal{F}_{\max }, z \in \mathbb{C}, u f(-1)+v f(1)=0\right\} .
$$

Define the following two Krein spaces:

$$
\mathcal{K}_{0}:=L_{2, r}\left(-\frac{1}{2}, \frac{1}{2}\right), \quad \mathcal{K}_{1}:=L_{2, r}\left(-1,-\frac{1}{2}\right)[\dot{+}] L_{2, r}\left(\frac{1}{2}, 1\right) .
$$

Extending the functions in $\mathcal{K}_{0}$ and $\mathcal{K}_{1}$ by 0 onto the rest of $[-1,1]$, we can consider the spaces $\mathcal{K}_{0}$ and $\mathcal{K}_{1}$ as subspaces of $L_{2, r}$.

Then

$$
L_{2, r}=\mathcal{K}_{0}[\dot{+}] \mathcal{K}_{1}
$$

Assume that the functions $p$ and $r$ satisfy Conditions 4.1 and 5.1. Let $W_{0}$ be the operator constructed in Theorem 4.2 and let $W_{-1}$ be the operator constructed in Proposition 5.3 with $\mu=1$. Then properties (IC) in Theorem 4.2 and Proposition 5.3. imply that $\mathcal{K}_{0}$ and $\mathcal{K}_{1}$ are invariant under $W_{0}$ and $W_{-1}$. As we chose $\mu=1$, we have $\left(W_{-1} f\right)(-1)=f(-1)$ and $\left(W_{-1} f\right)(1)=f(1)$. Define

$$
W_{01}:=\left.\left.W_{0}\right|_{\mathcal{K}_{0}}[\dot{+}] W_{-1}\right|_{\mathcal{K}_{1}} .
$$

Since $W_{0}$ and $W_{-1}$ are bounded, boundedly invertible and positive in the Krein space $L_{2, r}$, so is the the operator $W_{01}$. Also, $W_{01} \mathcal{F}_{\max }[-1,1] \subset \mathcal{F}_{\max }[-1,1]$ and

$$
\left[\begin{array}{c}
\left(W_{01} f\right)(-1) \\
\left(W_{01} f\right)(1)
\end{array}\right]=\left[\begin{array}{c}
f(-1) \\
f(1)
\end{array}\right]
$$

If the functions $p$ and $r$ satisfy Conditions 4.1 and 5.2 then, instead of $W_{-1}$, we use the operator $W_{+1}$ constructed in Proposition 5.4 with $\mu=-1$. Redefining the operator $W_{01}$ as

$$
W_{01}:=\left.\left.W_{0}\right|_{\mathcal{K}_{0}}[\dot{+}] W_{+1}\right|_{\mathcal{K}_{1}}
$$

we see that it is again bounded, boundedly invertible, and positive in the Krein space $L_{2, r}, W_{01} \mathcal{F}_{\max }[-1,1] \subset \mathcal{F}_{\max }[-1,1]$ and (since we use $\mu=-1$ )

$$
\left[\begin{array}{c}
\left(W_{01} f\right)(-1) \\
\left(W_{01} f\right)(1)
\end{array}\right]=-\left[\begin{array}{c}
f(-1) \\
f(1)
\end{array}\right]
$$


Now a simple inspection shows that, in both above cases, the operator

$$
W=\left[\begin{array}{cc}
W_{01} & 0 \\
0 & \Delta
\end{array}\right]: \begin{array}{ccc}
L_{2, r} & & L_{2, r} \\
& \mathbb{C}_{\Delta}
\end{array} \rightarrow \begin{gathered}
\oplus \\
\mathbb{C}_{\Delta}
\end{gathered}
$$

is bounded, boundedly invertible and positive in the Krein space $L_{2, r} \oplus \mathbb{C}_{\Delta}$ and $W \mathcal{F}(A) \subset \mathcal{F}(A)$. Thus the theorem again follows from Theorem 2.2.

Our final result covers the remaining cases, but in the interests of presentation we shall impose no conditions on L. Of course, there is some overlap with Theorem 6.1

Theorem 6.3. Assume that the following two conditions are satisfied.

(a) The coefficients $p$ and $r$ satisfy Condition 4.1, and

(i) Condition 5.1 if $\Delta>0$,

(ii) Condition 5.2 if $\Delta<0$.

(b) $\mathrm{N}_{n}=0$.

Then there is a basis for each root subspace of $A$, so that the union of all these bases is a Riesz basis of $L_{2,|r|} \oplus \mathbb{C}_{|\Delta|}$.

Proof. In this case (2.9) shows that the form domain of $A$ is

$$
\mathcal{F}(A)=\left\{\left[\begin{array}{c}
f \\
\mathrm{~N}_{e} \mathbf{b}_{e}(f)
\end{array}\right] \in \underset{\mathbb{C}_{\Delta}}{\stackrel{L_{2, r}}{\oplus}}: f \in \mathcal{F}_{\max }\right\}
$$

if $\mathrm{L}_{n} \neq 0$, and

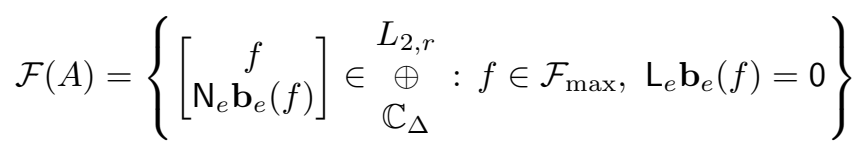

by (2.10) if $\mathrm{L}_{n}=0$.

Let $W_{01}: L_{2, r} \rightarrow L_{2, r}$ be the operator constructed in the proof of Theorem 6.2. and define $W: L_{2, r} \oplus \mathbb{C}_{\Delta} \rightarrow L_{2, r} \oplus \mathbb{C}_{\Delta}$ by

$$
W=\left[\begin{array}{cc}
W_{01} & 0 \\
0 & \operatorname{sgn}(\Delta)
\end{array}\right] .
$$

As before, in both cases the properties of $W_{01}$ imply that $W$ is a bounded, boundedly invertible, positive operator in the Krein space $L_{2, r} \oplus \mathbb{C}_{\Delta}$ and $W \mathcal{F}(A) \subset$ $\mathcal{F}(A)$. Now the theorem follows from Theorem 2.2

Remark 6.4. It is instructive to look at the above results from the viewpoint of non-essential boundary conditions $\left(\mathrm{L}_{n}, \mathrm{~N}_{n} \neq 0\right.$ ) and essential ones (whose essential parts $\mathrm{L}_{e}, \mathrm{~N}_{e}$ can be separated or not). Let us call a boundary condition essentially separated if it is either non-essential, or else its essential part is separated. Theorem 6.1 states that if both boundary conditions are essentially separated, then subject to the sign conditions in (CG-ii) and (CG-iii), Condition 4.1 suffices for the existence of a Riesz basis of root vectors. 
If any of these assumptions fail, then we impose conditions from Section 5. In particular, if the $\lambda$-dependent boundary condition is non-essential, then either of these conditions suffice, but in other cases the choice is governed by the sign of $\Delta$.

We conclude with a simple example.

Example 6.5. We suppose that

$$
\mathrm{L}=\left[\begin{array}{llll}
d_{1} & d_{2} & d_{3} & d_{4}
\end{array}\right], \quad \mathrm{M}=\left[\begin{array}{llll}
m_{1} & m_{2} & m_{3} & m_{4}
\end{array}\right], \quad \mathrm{N}=\left[\begin{array}{llll}
0 & \gamma & 0 & 0
\end{array}\right],
$$

where $\left(d_{3}, d_{4}\right) \neq(0,0)$ and $\gamma m_{4}>0$. Note that the only $\lambda$-dependent term in (1.2) involves $f(1)$.

We calculate

$$
\mathrm{MQ}^{-1} \mathrm{~N}^{*}=-i \gamma m_{4}
$$

so by (2.1), $\Delta>0$. It follows from Theorem 6.1 with condition (b-i) that Condition 4.1 suffices for the existence of a Riesz basis of root vectors.

This example overlaps with [11, Corollary 3.8], where separated boundary conditions, also satisfying $d_{2}=d_{4}=m_{1}=m_{3}=0, d_{3}=m_{4}=1, \gamma>0$, were considered by Fleige for a Krein-Feller equation instead of (1.1).

\section{Acknowledgment}

We thank a referee for a very careful reading of the submitted version of this article. This has led to the correction of a number of inaccuracies.

\section{References}

[1] N. L. Abasheeva, S. G. Pyatkov, Counterexamples in indefinite Sturm-Liouville problems. Siberian Advances in Mathematics. Siberian Adv. Math. 7 (1997), 1-8.

[2] R. Beals, Indefinite Sturm-Liouville problems and half-range completeness. J. Differential Equations 56 (1985), 391-407.

[3] P. A. Binding, B. Ćurgus, Form domains and eigenfunction expansions for differential equations with eigenparameter dependent boundary conditions. Canad. J. Math. 54 (2002), 1142-1164.

[4] P. A. Binding, B. Ćurgus, A counterexample in Sturm-Liouville completeness theory. Proc. Roy. Soc. Edinburgh Sect. A 134 (2004), 244-248.

[5] B. Curgus, On the regularity of the critical point infinity of definitizable operators. Integral Equations Operator Theory 8 (1985), 462-488.

[6] B. Ćurgus, H. Langer, A Krein space approach to symmetric ordinary differential operators with an indefinite weight function. J. Differential Equations 79 (1989), $31-61$.

[7] B. Ćurgus, B. Najman, Quasi-uniformly positive operators in Krein space. Operator theory and boundary eigenvalue problems (Vienna, 1993), 90-99, Oper. Theory Adv. Appl., 80, Birkhäuser, 1995. 
[8] A. Dijksma, Eigenfunction expansions for a class of J-self-adjoint ordinary differential operators with boundary conditions containing the eigenvalue parameter. Proc. Roy. Soc. Edinburgh Sect. A 86 (1980), 1-27.

[9] A. Fleige, The "turning point condition" of Beals for indefinite Sturm-Liouville problems. Math. Nachr. 172 (1995), 109-112.

[10] A. Fleige, A counterexample to completeness properties for indefinite Sturm-Liouville problems. Math. Nachr. 190 (1998), 123-128.

[11] A. Fleige, Spectral theory of indefinite Krein-Feller differential operators. Mathematical Research, 98 Akademie Verlag, 1996.

[12] H. Langer, A. Schneider, On spectral properties of regular quasidefinite pencils $F-$ $\lambda G$. Results Math. 19 (1991), 89-109.

[13] A. I. Parfyonov, On an embedding criterion for interpolation spaces and application to indefinite spectral problems. Siberian Math. J. 44 (2003), 638-644.

[14] S. G. Pyatkov, Interpolation of some function spaces and indefinite Sturm-Liouville problems. Differential and integral operators (Regensburg, 1995), 179-200, Oper. Theory Adv. Appl. 102, Birkhäuser, (1998).

[15] H. Volkmer, Sturm-Liouville problems with indefinite weights and Everitt's inequality. Proc. Roy. Soc. Edinburgh Sect. A 126 (1996), 1097-1112.

Paul Binding

Department of Mathematics and Statistics, University of Calgary, Calgary, Alberta, Canada, T2N 1N4

e-mail: binding@ucalgary.ca

Branko Ćurgus

Department of Mathematics, Western Washington University, Bellingham, WA 98225, USA

e-mail: curgus@cc.wwu.edu 